\title{
ELECTROPHYSIOLOGICAL CORRELATES OF THE RESPONSE DECREMENT PRODUCED BY MECHANICAL STIMULI IN THE PROTOZOAN, STENTOR COERULEUS
}

\author{
DAVID C. WOOD*
}

Brain Research Laboratory, The University of Michigan, Ann Arbor, Michigan 43104

\section{SUMMARY}

The response pattern of Stentor coeruleus has been studied using repetitive mechanical stimuli which were applied to a limited body surface. The decrement in the probability of response was found to parallel that previously reported when more gross mechanical stimuli were employed. This result justified the employment of such localized stimuli in the determination of electrophysiological correlates of the response decrement.

Electrophysiological records show a decrease in the amplitude of the prepotential and an increase in the latency of the response during the course of repetitive stimulation. After considering possible complications resultant from changes in the amplitude of the diphasic potential and the relation of the stimulus probe to the animal, it was concluded that the potential changes noted do, in fact, indicate a change in receptor function is associated with the response decrement. This result was predicted on the basis of previously reported behavioral data.

\section{INTRODUCTION}

In the first paper of this series (Wood, 1970a), the contractile behavior of Stentor coeruleus was studied in response to repeated mechanical stimulation. It was observed that the probability of response to the initial stimuli in a stimulus series was much higher than the probability of response to subsequent stimuli, i.e., a decrement in the probability of response was noted. This decrement was parametrically characterized and some evidence was also collected which suggested that the decrement was attributable to a change in sensory function. However, this latter conclusion was based on rather indirect evidence and on the hypothetical division of the processes occurring within the animal into sensory and effector processes.

*Presently at the Department of Psychology, The University of Pittsburgh, Pittsburgh, Pennsylvania 15213. This work represents part of a dissertation submitted to the University of Michigan in partial fullfillment of the requirements for a Ph.D. degree. 
In the second paper (Wood, 1970b), the contractions of Stentor were found to be correlated with the presence of a transient negative-going or diphasic response dependent on whether the recording electrode was intracellular or encapsulated respectively. Sufficient evidence was collected to conclude that the temporal sequence: mechanical stimulus $\rightarrow$ prepotential $\rightarrow$ spike potential $\rightarrow$ contraction provides a rudimentary model of the events and processes involved when mechanical stimuli are presented to Stentor.

It is obvious that the temporal sequence generated on the basis of the electrophysiological data is consistent with the previously suggested "receptor-effector" model used to analyze the behavioral data. One need only assume that the receptor process is represented by the prepotential in the electrophysiological records while the effector process involves the spike potential and subsequent chemical events leading to the contractions.

If it is assumed that this type of analogy exists between the models, then the electrophysiological correlate of the response decrement can be inferred from the behavioral data. Previously it has been proposed that the behavioral response decrement is primarily due to a modification in receptor function and not to a change in effector processes. In the electrophysiological records such a change should appear as a decrement in the prepotential without any alteration in the amplitude of the spike potential. The decrement in the prepotential would be expected to continue with repeated stimuli until finally no spike potential was produced.

The following experiments were designed to test this hypothesis. The design of the experiments was very simple and limited owing to the difficulty of maintaining stable microelectrode penetrations. A $1 / \mathrm{min}$ mechanical stimulus was applied to an animal penetrated with a micro-electrode until that animal failed to respond to the stimulus. The potential records were then analyzed for progressive changes in the recorded potentials and durations.

\section{METHODS}

Apparatus. The apparatus and method of culturing Stentor has been previously described (Wood, 1970a,b). All electrophysiological records were obtained from animals penetrated by only one microelectrode.

Procedure. In the first experiment reported here the response of animals mechanically stimulated with the piezoelectric crystal (phonograph cartridge) and blunted microelectrode was studied as a function of trials. Animals were placed in the test chamber at least $30 \mathrm{~min}$ prior to the initiation of stimulation to provide time for them to recover from mechanical stimulation associated with their being transferred into this chamber. The stimulating probe was then positioned adjacent to the side of the animal near its anterior end (see Fig. 1, Wood, 1970b). This area of the animal, including or adjacent to the membranellar band, appeared to be the most sensitive to mechanical stimuli. The stimulation probe was slowly moved to a position sufficiently close to the animal to produce a slight dimple in its pellicle.

Stimuli applied to different animals varied in amplitude but did not exceed a $0.25 \mathrm{~mm}$ displacement of the stimulus probe. At the beginning of a testing session the minimal 


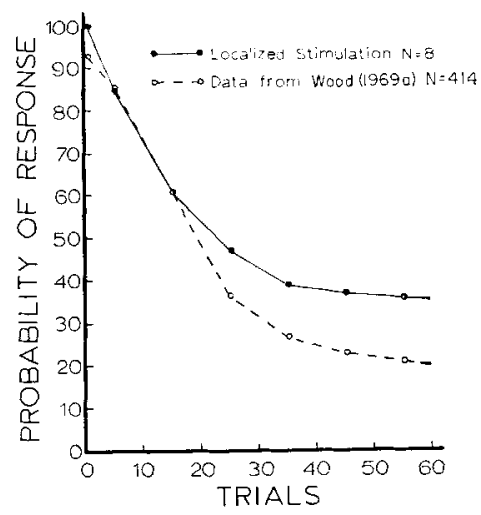

Fig. 1. Probability of response as a function of trials comparing the data when animals were stimulated with the piezoelectric crystal and stimulating probe and the data previously reported (Wood, 1970a).

of 3 amplitudes of mechanical stimuli (corresponding to potential differences of 40,60 , and $80 \mathrm{~V}$. across the piezoelectric crystal) which would cause an animal to contract was determined. After several minutes this stimulus was reiteratively employed during the subsequent $60 \mathrm{~min}$ at a rate of $1 / \mathrm{min}$. Because of this procedure all animals did not receive stimuli of the same amplitude; however, differences in the area of the animal stimulated the orientation of the animal and proximity of the stimulating probe meant that the effective stimulus intensity varied for different animals in any case.

If an animal contracted to a stimulus it was quite likely that it would reextend with a somewhat altered orientation with respect to the stimulus probe. This might have resulted in a change in the effective amplitude of the stimulus applied to the animal. To avoid this possibility the animal's original orientation was restored by slowly shifting its holdfast or anterior end in the proper direction by moving the animal with the stimulus probe which was mounted on a micromanipulator. This change was accomplished by a very slow motion to which the animals in this study did not respond. In all cases the stimulus probe was advanced so that it just dimpled the side of the animal prior to each stimulus.

In the second experiment both mechanical stimulation and microelectrode recording were employed. Animals were introduced into the test chamber $30 \mathrm{~min}$ before being penetrated with a microelectrode through the frontal field. During this $30 \mathrm{~min}$ animals became extended in the methyl cellulose suspension but did not become attached to the bottom of the test chamber. Successful penetrations were characterized by an initial shift in steady potential and small rippling fluctuations in potential similar to those previously reported (Wood, 1970b). The resistance in series with the electrode was also found to have increased.

After penetration 5 min were allowed to pass before stimulation was begun. In this period the encapsulation process apparently went to completion since all obtained records were of the diphasic form.

At the end of this rest period, the first of the mechanical stimuli was delivered by means of the blunted probe and piezoelectric crystal. The initial stimulus was produced by a $60 \mathrm{~V}$ pulse across the piezoelectric crystal or by an $80 \mathrm{~V}$ pulse if a larger pulse had been found necessary to produce contractions in animals studied previously in the testing session. If an animal failed to respond to the $60 \mathrm{~V}$ stimulus, an $80 \mathrm{~V}$ stimulus was used after an additional $3 \mathrm{~min}$ rest. The stimulus employed approximated that which was minimal to produce contractions; hence, few stimuli were required before the animal failed to respond to one, and no damage produced by the stimulus was 
evident. Once the stimulus amplitude required to produce contractions had been found this stimulus was reiteratively employed at a rate of $1 / \mathrm{min}$ until the animal failed to respond to it.

As in the previous experiment the relation of the stimulus probe to the animal was maintained constant by reorienting the animal with the stimulus probe. Each stimulus was delivered with the stimulus probe just touching the animal.

After an animal first failed to respond to a stimulus, $3 \mathrm{~min}$ were allowed to elapse in which no stimuli were applied. During this time no reorientation of the animal or stimulus probe was made. After this period another stimulus was applied. It was expected that during this 3 minute period partial recovery of responsiveness would be obtained if the preceding failure to respond were related to the previously studied decrement in response probability. $80 \%$ of the animals responded to the stimulus presented after the 3 min rest.

Finally, the animal was stimulated with an anodal current pulse of $1 \mathrm{msec}$ duration and less than or equal to $15 \times 10^{-8} \mathrm{amp}$. This procedure provided a check that the tested animal was actually impaled on the electrode since it was previously observed that impaled animals contract to such electrical stimuli (Wood, 1970b).

\section{RESULTS}

Eight animals were used in testing the pattern of response produced by repetitive localized stimulation. Of these 4 were stimulated by the probe excursion produced when a $40 \mathrm{~V}$ pulse was produced across the piezoelectric crystal, 3 were stimulated as a result of a $60 \mathrm{~V}$ pulse and 1 was stimulated as a result of an $80 \mathrm{~V}$ pulse. The point of stimulation varied for different animals though all were stimulated in close proximity to the membranellar band. One animal was stimulated on the membrane surface above the gullet, while 3 were stimulated on the diametrically opposed surface. Three were stimulated in an area near the contractile vacuole and 1 was stimulated on the side opposite this (see Fig. 1 of Wood, 1970b).

All 8 animals responded less frequently to the final 10 stimuli than to the initial 10 stimuli, thus a decrement in response probability was produced in all animals. The pattern of response probabilities against trials for these 8 animals is displayed in Figure 1. For comparison the response probabilities displayed in Figure 1 of Wood (1970a) are also included. Differences between these curves would be expected as a result of differences in subcultures, medium in the testing chamber, times of rest prior to stimulation, stimulus intensity, etc., as well as because of the difference in mode of stimulus presentation. Despite these several differences the patterns of response decrement are remarkably similar. This similarity suggests that the same decrement processes which occurred when the entire test chamber was moved also occur in response to more localized stimulation.

Since mechanical stimuli delivered by means of the piezoelectric crystal appeared to produce a response decrement similar to that studied previously, it became possible to study the electrophysiological correlates of this decrement by applying the more localized stimuli. In the study of these electrophysiological correlates, microelectrode recordings were obtained from 99 animals. 
It was impractical to attempt to record all the responses of animals given 60 stimuli at a rate of $1 / \mathrm{min}$. The fact that animals could and did become detached from the electrode prevented the use of such long recording sessions. Therefore, the recording was continued only until the animal failed to respond to a stimulus, that is to the first overt sign of a response decrement. In the median case reported below, this failure occurred after 6 (4-10) stimuli; whereas in the first experiment reported 7 (2-32) stimuli were applied before the first non-response.

Only 6 of the 99 records were judged satisfactory for final analysis, the remainder were discarded for failing to meet one of the several criteria listed below:

1) Only records showing diphasic spike potentials in excess of $10 \mathrm{mv}$ were used for analysis. Since the prepotential and first potential of the diphasic response were much smaller than the peak response, considerable percentage inaccuracy would have been involved in the quantification of these potentials in records showing spike potentials of less than $10 \mathrm{mv}$. Only 34 of the records met this criterion.

2) Since the response decrement is dependent on stimulation during preceding trials, it was felt advisable to study only those records in which there were a sufficient number of responses prior to the first non-response to allow some analysis of response amplitudes during the course of trials. It was therefore decided that only those records in which there were 4 or more responses before the first non-response would be analyzed. $21 \mathrm{rec}-$ ords met this criterion.

3) With the passage of time and repeated stimulation and contraction, the animals often became partially or completely separated from the electrode which resulted in diminution of recorded potential. Such changes could be confounded with changes correlated with the response decrement; therefore, it was decided to accept only records in which the amplitude of the second potential of the diphasic spike produced by the stimulus applied after the $3 \mathrm{~min}$ rest was 70 to $130 \%$ of its initial amplitude. The employment of this criterion placed certain arbitrary constraints on the data which will be discussed in subsequent pages. 8 records met this criterion.

4) Of these 8 records 6 had potential patterns similar to Figure $4 a$ of Wood $(1970 \mathrm{~b})$, while one resembled Figure $4 \mathrm{~b}$ and another resembled Figure 4c. Only the 6 animals showing the clear diphasic potential pattern were studied in this analysis.

The complete record of one of these penetrations is displayed as Figure 2. This animal contracted to 10 stimuli before first failing to contract. The record of the potentials correlated with the appearance of these contractions shows considerable variability in all the potential amplitudes recorded. For instance the second phase of the diphasic potential is maximal in the first frame with an amplitude of $18.0 \mathrm{mv}$ and minimal in the third frame with an amplitude of $11.9 \mathrm{mv}$. The amplitude of the first diphasic potential shows even greater percentage variability having a 


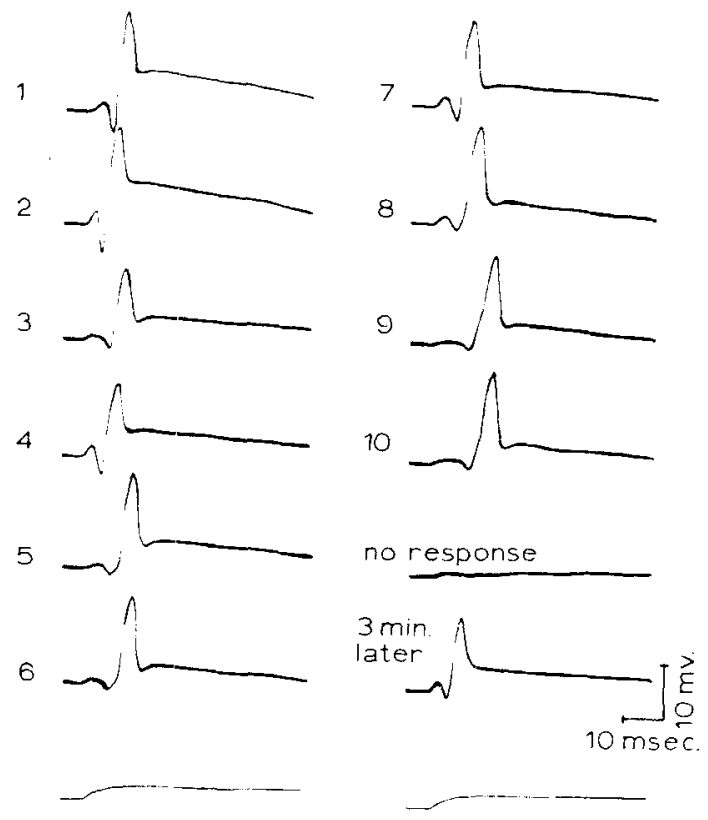

Fig. 2. A sample series of spike potentials recorded during a series of mechanical stimuli presented at $1 / \mathrm{min}$. The bottom trace of each column represents the electrical pulse which actuated the mechanical stimulus. This figure is a tracing.

value of $6.8 \mathrm{mv}$ in the second frame and only $1.1 \mathrm{mv}$ in the ninth frame. As predicted the prepotential shows an irregular but progressive decrease in amplitude from $1.6 \mathrm{mv}$ in frame 1 and $2.1 \mathrm{mv}$ in frame 2 to $0.5 \mathrm{mv}$ in frames 9 and 10 . The spike potential occurring after the $3 \mathrm{~min}$ rest shows recovery in the amplitude of both these latter parameters. A slight graded potential is observable in the record of the nonresponse trial.

To analyze these records the amplitude of the prepotential and second phase of the diphasic spike were measured above the steady potential observed before stimulation. The amplitude of the first phase of the diphasic spike was measured from its apparent point of onset to its peak. This latter measure was chosen because it was felt the entire diphasic potential might ride on a sustained grade potential such as that seen in Figure 8 of Wood $(1970 \mathrm{~b})$. Measuring either the first phase of the diphasic spike from the baseline or the second phase of the spike from the peak of the graded potential does not alter the analysis; if anything the results then show less variability and less change in the amplitude of these potentials with trials.

The combined data from the 6 animals are presented as Figure 3. The amplitude of the various potentials are plotted as a percentage of their amplitude during the first block of trials to make changes in the small amplitudes of the prepotential and first phase of the diphasic spike visible 


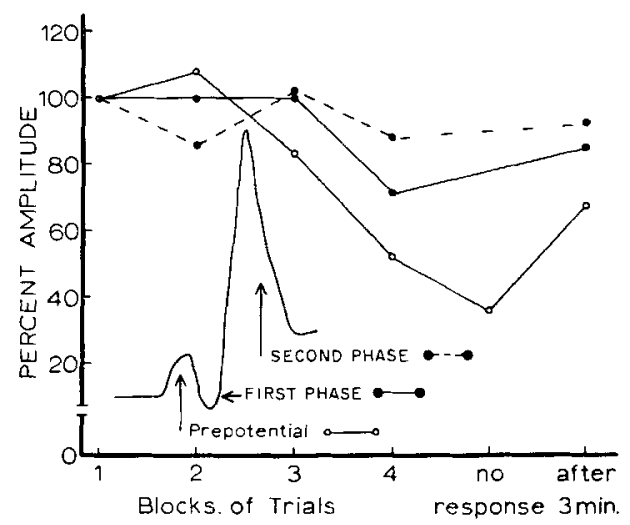

Fig. 3. Percent potential amplitudes as a function of trials. Measurements as described in the text.

in comparison with the changes in the large amplitude second phase. The number of trials per block was determined by distributing the trials as evenly as possible among the four blocks and then randomly assigning remaining trials to one of the four blocks. The amplitude of the graded potential during the non-response trial was also measured as were the parameters of the response obtained after the 3 min rest period.

The most obvious change isthe decrement in the amplitude of the prepotential with trials. The mean percent amplitude of the prepotential during block IV was significantly different from the block I mean $(p<$ 0.001 , t test) for matched samples (Underwood, Duncan, Taylor and Cotton, 1954). The amplitude of the graded potential observed during the non-response trial is also significantly different from the $100 \%$ level.

These data are consonant with the view that the prepotential decreases over the course of trials while the other recorded potentials remain essentially unaltered. However, both peaks of the diphasic potential were depressed somewhat during the fourth block of trials; therefore, the decrease in prepotential amplitude might be attributable to a general depression of all potential amplitudes. The percent amplitude of the prepotential was, however, significantly less than the percent amplitude of the first phase of the diphasic response during block IV $(p<0.05, t$ test $)$ and the percent amplitude of the second phase during this same block of trials $(\mathrm{p}<0.02, \mathrm{t}$ test). It is therefore possible to conclude that relative to the other recorded potentials the amplitude of the prepotential was depressed.

The depression of the prepotential amplitude was observed in all 6 records reported here, in the 2 records of animals whose recorded diphasic response was of a somewhat different form, and in several records where animals responded only 3 times prior to the first non-response. There were no recorded exceptions to this observation. 
D. C. WOOD

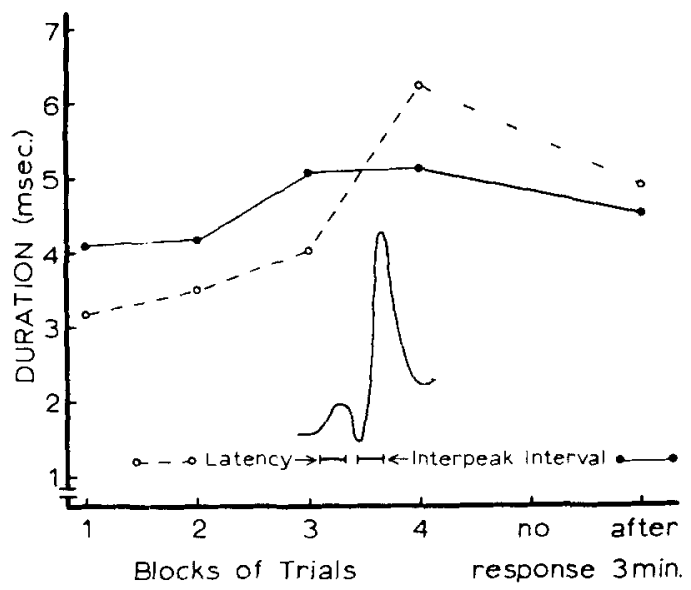

Fig. 4. Latency of response and interpeak interval as a function of blocks of trials. Data derived from the same records as the data incorporated into Figure 3 . Measurements taken as described in text and indicated in the figure.

Perhaps even more pronouced than the decrease in prepotential amplitude was the increase in latency observed in most records and evident in Figure 2. To quantify this change temporal measurements were made from the onset of the prepotential to the onset of the diphasic response. The onset of the stimulus pulse was not used as the point of reference so that time required to initial probe movement and for the probe to contact and affect the animal would not be involved in the measurements. As a control measure the time between the peaks of the diphasic response was also quantified. Data was grouped into blocks as previously described.

Figure 4 displays the results of this analysis. The response latency in the third block of trials is significantly different from that of the first block of trials ( $p<0.02$, t test). Likewise, the latency in block IV is significantly different from the latency of block $I(p<0.05, t$ test). A hint of this change can be seen in the sample record (Fig. 2). As trials progressed there was some tendency for records to show slight irregularities and subpeaks. A possible explanation of this change may be that the recorded potential is actually a composite and that several components of the potential can show temporal shifts relative to one another.

Since latencies can be recorded accurately even from relatively small potentials, it was possible to analyze temporal intervals on 12 records where the response amplitude was less than $10 \mathrm{mv}$. However, in these cases the latency had to be measured from the stimulus onset since the prepotential onset was often not discernible. In these cases (Fig. 5) there is also a clear and pronounced increase in response latency; the latency of response during block IV trials is significantly different from the latency of block I trials ( $p<0.02$, t test). However, no change was evident in the interval between the diphasic spikes. 


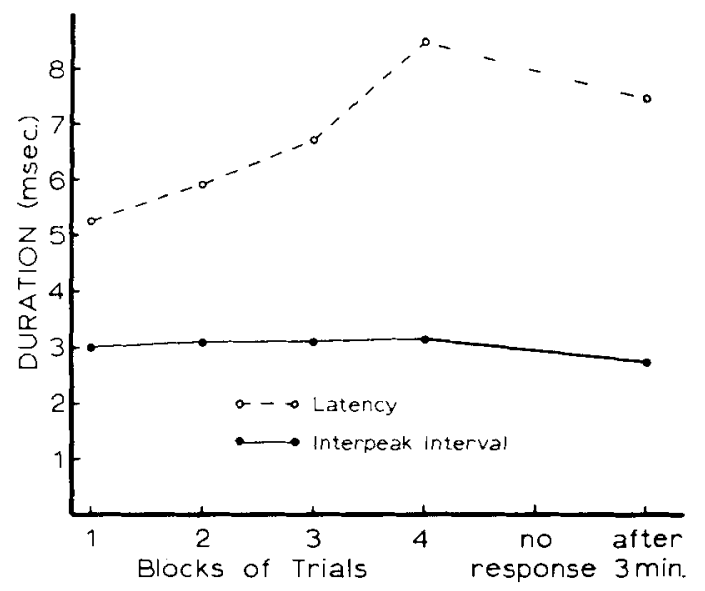

Fig. 5. Latency of response and interpeak interval as a function of blocks of trials. Measurements taken on 12 animals as described in text.

It can be concluded that the latency of the diphasic response did increase with trials while the interpeak interval may or may not have changed.

\section{DISCUSSION}

In the first experiment reported here it was found that localized mechanical stimulation produced a response decrement of similar time course to that observed when more general stimulation was applied. Localized stimulation was employed in the second experiment to ascertain what electrophysiological changes occur during the initial phase of the response decrement. A depression of the prepotential relative to the other recorded potentials and an increase in response latency were the only consistent and pronounced changes noted. It is always possible that other changes may have occurred, such as an increase in the interpeak interval or a decrease in the amplitude of the first phase of the diphasic response, and that the sample size or method of analysis was insufficient to reveal these changes.

Only records in which the second phase of the diphasic response returned to $70 \%$ of its initial amplitude after the 3 min rest interval were deemed satisfactory for further analysis. This selection criterion was necessary because decrements in potentials were observed with time and stimulation apparently resulting from the animals becoming partially disengaged from the electrode. In such cases electrical stimulation delivered via the electrode failed to produce contractions and extended rests $(10 \mathrm{~min}$ ) failed to restore the amplitude of the potentials recorded. Because of this criterion a decrease in the amplitude of the second phase beyond $70 \%$ was unlikely to be observed during the stimulation series. In fact this limit was not approached, nor was there any consistent trend 
in the amplitude of this potential over trials. It therefore seems likely that the employment of the $70 \%$ criterion did not seriously alter the results.

Both the decrease in the prepotential amplitude and the increase in latency could have been produced by a progressive decrease in stimulus intensity. Since the excursion of the stimulating probe was held constant through the series of trials the only change possible in the stimulus was an alteration in the relation between the stimulus probe and animal. Specific care was taken to prevent such alterations by reorienting the stimulus probe after each contraction under direct visual control. However, certain other evidence suggests that the electrophysiological changes noted were not a result of a decrease in stimulus intensity. Firstly, latencies for the diphasic potential responses were measured from the onset of the prepotential rather than the onset of the stimulus; hence, the change in latency reflected a change in the effectiveness of the stimulus actually operating on the animal. Changes in the time for the stimulus to act on the animal attributable to changes in the distance between the probe at rest and the animal did not enter into these data. Secondly, there was no reorientation of the stimulus probe during the $3 \mathrm{~min}$ rest period; hence, the stimulus probe was generally farther from the animal on this trial than during block IV trials. Nevertheless, both the prepotential amplitude and response latency observed after the $3 \mathrm{~min}$ rest show considerable recovery from the block IV values. In sum, there are several lines of evidence suggesting the changes in latency and prepotential were not resultant from a progressive diminution of the effective stimulus.

It therefore appears that the electrophysiological changes noted are in agreement with those predicted on the basis of the behavioral data reported earlier. The decrease in prepotential amplitude and increase in response latency with trials both imply that one, and presumably the principal, process which accompanied the response decrement was a decrement in receptor function.

In a variety of biological preparations neural response decrements correlated with habituation of overt behaviors have been observed (Horridge, 1959; Roberts, 1962; Roeder, 1962). In all these cases these decrements have been attributed primarily to decrements in synaptic transmission. In studies where habituation has been analyzed for specific neural correlates, the data have suggested that two different mechanisms may be operative. Bruner and Tauc (1966) and Spencer, Thompson, and Neilson (1966) have reported that a depression of EPSP amplitude is correlated with habituation of neural response, while Holmgren and Frenk (1961) and Kandel (1967) observed a recruitment of IPSP's in their habituating preparations. The process observed in Stentor might possibly be analogous to an EPSP depression if the later is due to an alteration of the postsynaptic neural membrane. On the other hand, Bruner and Tauc presented evidence suggesting a presynaptic membrane change was most likely in their preparation. Nevertheless, in broad out- 
line the phenomenon noted in Stentor parallels that noted in some metazoan preparations; in both instances a depression of the prepotential appears to be the principle process. Whether the analogy can be extended further depends on whether this effect is a change in pre- or postsynaptic elements in the metazoan and on similarities or differences in biochemical processes which have not yet been studied.

\section{REFERENCES}

Bruner, J. and Tauc L. (1966). Habituation at the synaptic level in Aplysia. Nature 210: 37-39.

Holmgren, B. and Frenk, S. (1961). Inhibitory phenomena and "habituation" at the neuronal level. Nature 192: 1294-1295.

Horridge, G. A. (1959). Analysis of the rapid responses of Nereis and Harmothoe (Annelida). Proc. Roy. Soc. B. 150: 245-262.

Kandel, E. R. (1967). Cellular studies of learning. In The Neurosciences: a study program. I. C. Quarton, T. Melnechuk and F. O. Schmitt (eds), Rockefeller University Press, New York.

Roberts, M. B. V. (1962). The giant fibre reflex of the earthworm, Lumbricus terrestris L: II. Fatigue, J.exp. Biol. 39: 229-237.

Roeder, K. O. (1962). Neural mechanisms in animal behavior. Am. Zool. 2: 105-115.

Spencer, W. A., Thompson, R. F. and Neilson, Jr., D. R. (1966). Decrement of ventral root electrotonus and intracellularly recorded PSPs produced by iterated cutaneous afferent volleys. J. Neurophysiol. 29: 253-274.

Underwood, B. J., Duncan, C. P., Taylor, J. A. and Cotton, J. W. (1954). Elementary statistics. Appleton-Century-Crofts, New York.

Wood, D. C. (1970a). Parametric studies of the response decrement produced by mechanical stimuli in the protozoan, Stentor coeruleus. J. Neurobiol. 1: 345-360.

Wood, D. C. (1970b). Electrophepiological studies of the protozoan, Stentor coeruleus. J. Neurobiol. 1: 363-377.

The author would like to gratefully acknowledge the material support provided him by $\mathrm{Dr}$. James Olds during the course of these studies. He is also greatly indebted to Dr. James B. Ranck, Jr. for his suggestions during the course of experimentation and writing of the manuscript.

During the course of these studies the author was supported by a predoctoral fellowship provided by the N. I. H. 